\title{
COVID-19 features in children and adolescents: a systematic review and pooled analysis
}

Eleni D. Panagouli ${ }^{1}$, Athanasios Ch. Thirios ${ }^{1}$, Theodora Psaltopoulou ${ }^{2}$, Flora Bacopoulou ${ }^{3 \otimes}$, Theodore G. Troupis $^{4}$, George P. Chrousos ${ }^{3}$, Maria N. Tsolia ${ }^{1}$, Theodoros N. Sergentanis ${ }^{1}$, Artemis K. Tsitsika ${ }^{1}$

\author{
'Adolescent Health Unit, Second Department of Pediatrics, "P. \& A. Kyriakou" Children's Hospital, National and Kapodistrian \\ University of Athens, Greece \\ 2Department of Clinical Therapeutics, School of Medicine, National and Kapodistrian University of Athens, Greece \\ ${ }^{3}$ University Research Institute of Maternal and Child Health \& Precision Medicine, and UNESCO Chair on Adolescent Health Care, \\ National and Kapodistrian University of Athens, Aghia Sophia Children's Hospital, Greece \\ ${ }^{4}$ Department of Anatomy, Medical School, National and Kapodistrian University of Athens, Greece \\ Competing interests: EDP none; ACT none; TP none; FB none; TGT none; GC none; MNT none; TNS none; AKT none
}

\section{Abstract}

The purpose of the present study was to evaluate special features and probable correlations between symptoms, laboratory findings, treatment, and outcomes of COVID-19 in children and adolescents, through a systematic review and pooled analysis. Following database (Pubmed, Google Scholar, Scopus and Embase) search, forty articles were considered eligible identifying a total of 2,971 confirmed pediatric COVID-19 patients. Fever was reported in $55.1 \%$ of the cases, while $28.4 \%$ were asymptomatic. Radiological signs of pneumonia were observed in more than half of the cases and in $40.7 \%$ of asymptomatic patients. Fever showed the highest sensitivity (sensitivity: $60.3 \%$, specificity: $48.8 \%$ ), followed by cough (sensitivity: $47.4 \%$, specificity: $76.7 \%$ ), rhinorrhea (sensitivity: $21.1 \%$, specificity: $88.4 \%$ ) and diarrhea (sensitivity: $10.3 \%$, specificity: $88.4 \%$ ), in differentiating cases with positive radiological signs for pneumonia. Compared to school age children, preschoolers (adjusted OR=6.01, 95\%CI: 1.73-20.91) were more prone to pneumonia findings. Various combinations of treatments were used across studies, without following any strict guidelines. Most children $(>90 \%)$ had full recovery and rarely presented complications. Fever seems to be the most frequent symptom in pediatric COVID-19, but pediatricians should additionally evaluate cough, rhinorrhea, and diarrhea as indicators of SARS-CoV-2 infection. Asymptomatic cases were common, but not the majority, and a significant percentage had developed radiologic findings of pneumonia. Thorough reassessment of treatment and management guidelines should be helpful.

\section{Introduction}

Since December 2019, when there was the initial outbreak of the novel Coronavirus disease (COVID-19) (Zhu et al., 2020) and after its classification by the World Health Organization (WHO) as a pandemic, on 11 March, 2020, the world is struggling to solve the issues arising by Severe Acute Respiratory Syndrome Coronavirus 2 (SARS-CoV-2). The accumulating knowledge and experience, resulting from the numerous cases around the globe, overwhelms the medical community with information that needs to be critically synthesized and presented in a reliable way, so it can be helpful for physicians. By May 1, 2020, there have been more than 3 million confirmed cases and around 233,000 deaths due to COVID-19 (WHO, 2020). According to the literature,
$0.9 \%$ of the recorded cases were less than 9 years old, and $1.2 \%$ were aged between 10 and 19 years (WHO, 2020).

Although there is a number of studies ( $\mathrm{Hu}$ et al., 2020) reviewing characteristics and symptoms of COVID-19, they are principally referring to adult population. Most review studies are management recommendations (Chen et al., 2020; Kanne, 2020; Shen et al., 2020), based on previously acquired knowledge on SARS caused by other known coronaviruses. There are only few studies or case reports referring exclusively to children and adolescents, that describe with detail COVID-19 characteristics (Ludvigsson, 2020). This might be due to the fact that children and adolescents are more resilient to the disease (Ludvigsson, 2020), presenting with milder symptoms and have a very low mortality rate. Nevertheless, infants ( $0-1$ years) seem to

\section{Article history}

Received: 30 August 2020

Accepted: 29 September 2020

Published: 26 November 2021

(c) 2021 Panagouli et al.; the authors have retained copyright and granted the Journal right of first publication; the work has been simultaneously released under a Creative Commons Attribution Licence, which allows others to share the work, while acknowledging the original authorship and initial publication in this Journal. The full licence notice is available at http://journal.embnet.org. 
be more prone to every kind of infection. The majority of deaths have been described in the age group between 1019 years (Castagnoli et al., 2020). Thus, there is a need for a full description of the virus characteristics in children and adolescents, to guide early diagnosis and successful management.

Until now, there have been a few systematic reviews considering COVID-19 in children (Castagnoli et al., 2020; Chang et al., 2020; Ludvigsson, 2020). All of them have been based on a small number of reports, the majority of which are derived from China and often suffer from missing data. Castagnoli et al. (2020) have highlighted the need for further studies and evaluation in a greater sample. The purpose of the present study is to perform a systematic review and pooled analysis in order to evaluate correlations between symptoms, laboratory findings, treatment and outcome of COVID-19 in children and adolescents. Specifically, this study aims to evaluate sensitivity, specificity, positive predictive value (PPV) and negative predictive value (NPV) of symptoms predicting pneumonia in radiological exams, as well as the association of pneumonia radiological findings with sex and age of children and adolescents.

\section{Materials, Methodologies Techniques}

\section{Study Design}

The present systematic review and pooled analysis was performed according to the PRISMA guidelines (Liberati et al., 2009). For this review, the Pubmed, Google Scholar, Scopus and Embase databases were searched for eligible studies for the period up to 15 April 2020. The search was limited to this year's publications, as SARS-CoV-2 was first identified in December 2019. The following search algorithm was used: (Covid-19 OR SARS-CoV-19 OR SARS-CoV-2 OR "2019-nCoV” OR "novel coronavirus") AND (child OR children OR pediatric OR pediatrics OR kid OR kids OR youngster OR youngsters OR adolescent OR adolescents OR teen OR teens OR teenager OR teenagers). The references of all the articles that were considered eligible were also thoroughly checked.

\section{Inclusion criteria}

Only original research articles (cohort studies, crosssectional studies, clinical studies, and case series) and case reports written in English were included. The eligible studies should refer to the symptoms, laboratory findings, treatment and infection outcome in children and adolescents exposed to SARS-CoV-2. In order to avoid misleading data, studies with several data missing, or the infection was categorized into "types" without clearly mentioning the symptoms or several data were missing, were excluded. The reported patients should be under 18 years old with confirmed positive COVID-19 infection. No limitations concerning race, sex or journal were imposed. Overlapping populations and any type of unpublished material were excluded.

\section{Eligibility assessment and risk of bias as- sessment}

Two independent reviewers (E.P. and A.T.) performed the screening for eligibility of the retrieved studies. Concerning the risk of bias, the Newcastle-Ottawa Quality Scale (Stang, 2010) was used to evaluate the quality of the included studies. Studies that did not meet the eligibility criteria, studies containing incomplete or irrelevant data, or studies with risk of bias were excluded.

\section{Data extraction}

The two reviewers screened the studies that were considered eligible and extracted all relevant data. For each study, the following parameters were collected: Author and year of publication, country where the study was conducted, study design, study period, number of specimens, mean age and age range, gender, health condition of patients before infection, symptoms caused by COVID-19, laboratory findings and outcome of the disease. After the evaluation of data, results were tabulated. Case reports and case studies were categorized separately. All kinds of symptoms mentioned in every eligible study were recorded and infection severity was classified accordingly. Concerning laboratory findings, data were extracted about the total number of white blood cells (WBC), lymphocyte count, neutrophil count, C-reactive protein (CRP), procalcitonin (PCT), d-dimer and imaging findings, computed tomography $(\mathrm{CT})$ scan and where not available, X-rays.

\section{Statistical analysis}

All data were recorded in the form of tables and were subjected to statistical analysis (pooled analysis). For categorical variables, frequency and percentage were calculated, while the mean, range and standard deviation (SD) were calculated for continuous variables. Pearson's chi-squared test was used to evaluate associations between nominal variables. Univariate and multivariate logistic regression analysis was performed to evaluate gender and age as potential risk factors of pneumonia radiological findings; odds ratios (ORs) and 95\% confidence intervals (CIs) are reported. Kaplan-Meier curves were estimated for time to negativity in nasal/ throat swab and fecal tests by age groups and logrank tests were employed to examine any difference (Šimundić, 2009). Statistical analysis was performed with STATA/SE version 13 statistical software (Stata Corp., College Station, TX, USA).

\section{Results}

The database search produced 1,320 studies. After the removal of duplicates and the assessment of 401 items, a total of 40 articles (Zhang, 2020; Cai et al., 2020; Canarutto et al., 2020; Chan et al., 2020; CDC, 2020; Cui et al., 2020; Han et al., 2020; Z. Hu et al., 2020; Huang et al., 2020; Ji et al., 2020; Kam et al., 2020; Le et al., 2020; B. Li et al., 2020; W. Li et al., 2020; Li and Guo, 2020; 
Lin et al., 2020; H. Liu et al., 2020; M. Liu et al., 2020; Liu and Zhang, 2020; Y. Liu et al., 2020; Lou et al., 2020; Lu et al., 2020; Ma et al., 2020; Pan et al., 2020; Park and Han, 2020; Qian et al., 2020; Qiu et al., 2020; Robbins et al., 2020; Q. Shen et al., 2020; Sun et al., 2020; Tang et al., 2020; Wang et al., 2020; Wei et al., 2020; Xia and Shao, 2020; Xing et al., 2020; R. Xu et al., 2020; Y. Xu et al., 2020; Zhang et al., 2020; Zheng et al., 2020;Zhu et $a l ., 2020)$ were considered eligible. The steps regarding the selection of eligible studies are presented in Figure 1.

Among them, 33 were case reports and case studies (118 patients), which presented detailed characteristics of the patients involved, and seven were retrospective cohort studies (2,853 patients), in total 2,971 patients. Low risk of bias was observed for cohort studies; however, the short follow-up periods could represent a compromising factor for the quality of studies.

\section{Case reports and case series}

\section{Demographics}

Data of case reports and case series (33 in total) are presented in Tables 1 and 2 in Supplementary Data ${ }^{1}$. Most studies derived from China, but there were also cases from Italy, Vietnam, USA and Singapore; a total of 118 children positive for SARS-CoV-2 were recorded [61 (51.7\%) males and 57 (48.3\%) females]. Their age ranged between 2 days and 18 years (mean age \pm SD $6.0 \pm 4.8$ years). Only $13.5 \%$ of the sample were adolescents $(>12$ years). In most patients (97.4\%) the presence of SARSCoV-2 RNA was detected by real-time fluorescence reverse-transcriptase polymerase-chain reaction (RTPCR) either in nasopharyngeal and throat swabs or feces. Health status and comorbidity were not clearly mentioned in most studies. Nevertheless, the majority did not seem to have other health problems. Only one case of a 2-month-old male with respiratory syncytial virus (RSV), one case of a 7-year-old female with influenza, one case of an 8-year-old male with acute lymphoblastic leukemia, one case of a 10-monthold female with lacrimal sac dredge, and one case of a 9 -year-old male with tonsillitis were reported (Table 1 in Supplementary Data $\left.{ }^{1}\right)$.

\section{Clinical Symptoms}

The most frequently reported clinical finding of COVID-19 in children was fever $(55.1 \%$ of the cases, $64 / 116)$, combined or not with other symptoms, while $28.4 \%(33 / 116)$ of the cases were asymptomatic. Fever was the only symptom in $19.8 \%$ of the cases $(23 / 116)$. In a small percentage $(16.4 \%, 19 / 116)$, fever was absent. The most commonly recorded symptoms and signs in these cases were cough, sore throat, diarrhea, vomiting, rhinorrhea, tachypnea and crackles, especially in infants. In two studies the clinical symptoms were not recorded (1.7\%). It should be mentioned that all cases with
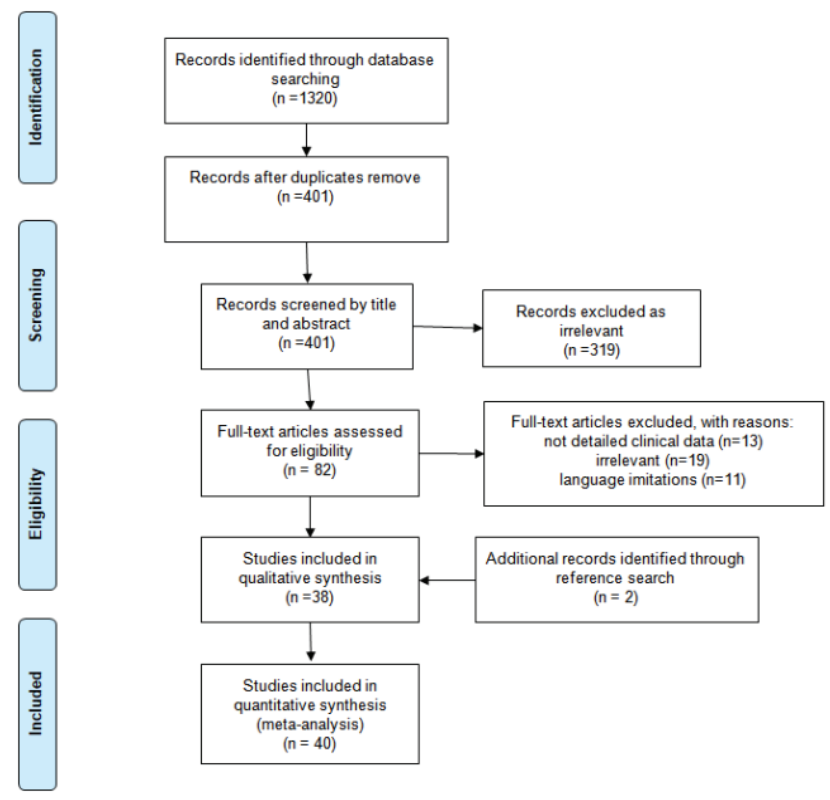

Figure 1. Prisma flow diagram.
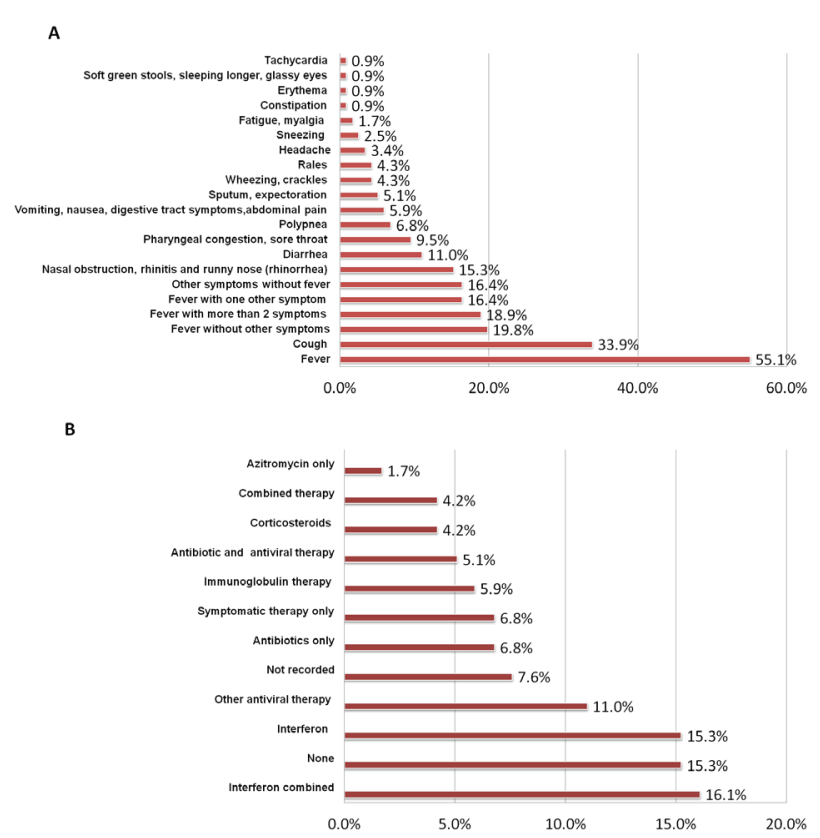

Figure 2. Diagrams - A. Symptoms in children positive with Covid-19. B. Distribution of administered drugs and treatments. Combined therapy: Antibiotic treatment and antiviral treatment and interferon (spray inhalation or nebulization) and glucocorticoids and intravenous immunoglobulin and symptomatic treatment. Corticosteroids: alone or combined with other therapies. Immunoglobulin therapy: alone or with antivirals and antibiotics or interferon (spray inhalation or nebulization). Other antiviral therapy such as lopinavir, ritonavir, ribavirin, oseltamivir, virazole. Interferon: spray inhalation or nebulization. Interferon combined: spray inhalation or nebulization, together with antibiotic, other antiviral or symptomatic therapy. 
tachypnea were admitted to intensive care unit (ICU) (Sun et al., 2020). There may be a potential association between asymptomatic status and male gender (63.6\% cases, Pearson's chi-squared $=3.01, \mathrm{p}=0.08)$. Clinical findings in children are represented in Figure 2A.

\section{Laboratory and radiological findings}

A detailed analysis of laboratory and radiological findings for each case is presented in Table 2 in Supplementary Data $^{1}$. Some of the included studies did not provide laboratory or radiological findings (14.4\%, 17/118). Less than $4 \%$ of cases presented deviations from the normal values of WBC, lymphocytes, neutrophils, CRP, PCT or d-dimer (Table 2 in Supplementary Data ${ }^{1}$ ). More than half of children $(57.4 \%, 58 / 101)$ had positive imaging findings (CT scans or X-rays) suggestive of unilateral or bilateral pneumonia. Interestingly, eleven of them were asymptomatic. The rest of the cases had normal CT scans or X-rays $(42.6 \%, 43 / 101) ; 64.3 \%$ of them $(27 / 43)$ presented various clinical symptoms and the rest were asymptomatic. It is worth mentioning that in 4 cases, elevated levels of aspartate aminotransferase (AST) and alanine aminotransferase (ALT) were recorded and that in 22 cases prolonged viral shedding was observed in the feces of patients (Table 2 in Supplementary Data ${ }^{1}$ ).

Time to negativity in nasal/throat swab RT-PCR test (Table 1 in Supplementary Data ${ }^{1}$ ), by age groups, is shown in the Kaplan-Meier curve of Figure 3; no significant difference was observed regarding age groups $(\mathrm{p}=0.43$, log-rank test) or gender $(\mathrm{p}=0.71, \log$-rank test). Similarly, no difference was noted in time to negativity of fecal tests by age $(\mathrm{p}=0.55)$ or gender $(\mathrm{p}=0.27)$.

\section{Gender and age as risk factors of pneu- monia}

As noted above, $57.4 \%$ of the patients eventually developed radiological findings suggestive of pneumonia. Table 3 in Supplementary Data ${ }^{1}$ presents the results of univariate and multivariate logistic regression analysis examining risk factors for such radiological signs. Compared to children aged 5 - 11 years, those aged 1 to 4 years presented with 6-fold increased odds of pneumonia findings in CT or X-ray (adjusted OR=6.01, 95\%CI: 1.73-20.91); adolescents also showed more frequently radiological images compatible with pneumonia (adjusted $\mathrm{OR}=2.52$, 95\%CI:0.79-8.03). Gender was not associated with pneumonia radiological findings (adjusted $\mathrm{OR}=0.75$, 95\%CI: 0.32-1.73).

\section{Sensitivity and specificity of symptoms to predict pneumonia}

The presence of symptoms predicted radiological pneumonia with a pooled sensitivity of $81.0 \%$ (47 out of 58 pneumonias, 95\%CI: $68.6-90.1 \%$ ) and a pooled specificity of $37.2 \%$ (16 out of 43 pneumonia-free cases, 95\%CI: 23.0-53.3\%). The pooled positive predictive value

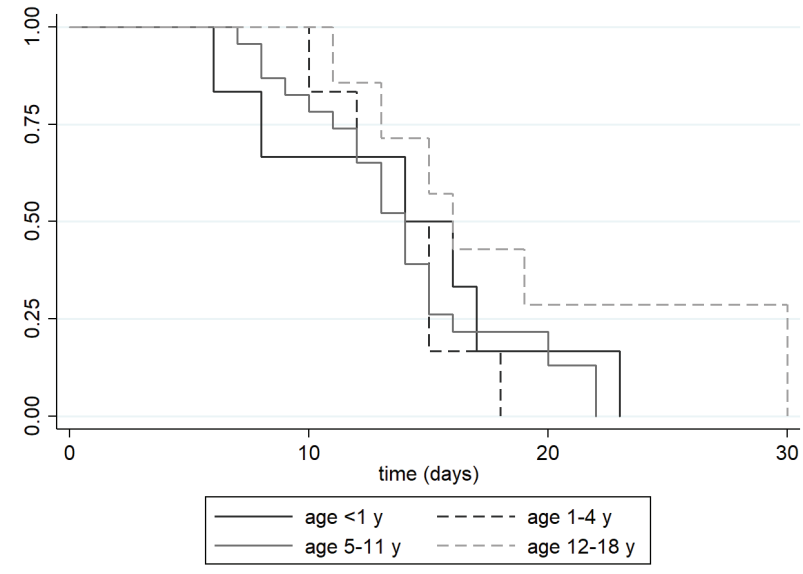

Figure 3. Kaplan Meier curve - Kaplan Meier estimates for negativity in nasal/throat swab RT-PCR test.

was $63.5 \%$ (95\%CI: $51.5-74.4 \%)$, whereas the pooled negative predictive value was $59.3 \%$ (95\%CI: 38.8-77.6\%).

Among individual symptoms, fever (sensitivity: $60.3 \%$, specificity: $48.8 \%$ ) was most common, followed by cough (sensitivity 47.4\%, specificity: $76.7 \%$ ), rhinorrhea (sensitivity: 21.1\%, specificity: $88.4 \%$ ) and diarrhea (sensitivity: $10.3 \%$, specificity: $88.4 \%$ ). On the other hand, rhinorrhea and diarrhea showed the highest specificity in differentiating cases with positive radiological signs for pneumonia from negative ones.

\section{Therapeutic management}

Multiple combinations of treatments were used in each study (Figure 2B). In a small percentage $(7.6 \%, 9 / 118)$ the treatment was not clearly recorded. Many cases, $83.5 \%$ (91/109), received therapy $24.2 \%$ (22/91) of which were asymptomatic. The remaining $16.5 \%$ (18/109) received no therapy at all. Most common treatments were symptomatic (antipyretics, oxygen therapy, vitamin $\mathrm{C}$ and Montelukast sodium chewable tablets). Alternative therapies were also applied, such as traditional Chinese medicine and oral probiotics. Antiviral therapy, such as lopinavir (9/109), ritonavir (9/109), ribavirin (14/109), oseltamivir (16/109), virazole (8/109), was mainly administered orally, while interferon was applied as a spray for inhalation or nebulization. Azithromycin was the most frequently used antibiotic (6/109), others being ceftriaxone (2/109), moxifloxacin (1/109), and penicillin G (1/109). Patients in ICU received combined therapies together with hemopurification, enterostomy, transfusions of red blood cells, plasma and thrombocytes (1 case) and plasmapheresis (1 case). It should be noted that most studies referred to therapies generally as "antiviral" or "antibiotics", without providing further details.

\section{Outcome}

Missing data concerning the outcome of pediatric COVID-19 were acknowledged in 6 cases (5.1\%). 
Most children had full recovery and presented no complications (105/112, 93.7\%). Among them, 68.7\% (77/112) were hospitalized, of which $24.6 \%$ (19/77) were asymptomatic. Only nine children (age range $2 \mathrm{~m}$ to 15 years, mean \pm SD $7.3 \pm 5.8$ years) were admitted to ICU (8.0\%), among them the patient with acute leukemia whose infection was surprisingly uncomplicated. No significant differences were observed between males and females concerning hospitalization (Pearson's chisquared=1.06, $\mathrm{p}=0.30$ ) and admission to ICU (Pearson's chi-squared=0.77, $\mathrm{p}=0.38$ ). Radiological findings of pneumonia were strongly associated with admission to ICU (9/54 children with pneumonia were admitted to ICU vs. 0/41 without pneumonia, $\mathrm{p}=0.009$, Fisher's exact test). Complications were recorded in $3.6 \%(4 / 112,3$ males and 1 female) of cases, leading to ICU admission. No deaths were reported.

\section{Retrospective cohort studies}

Demographic data about retrospective cohort studies (7 in total) are presented in Table 1 in Supplementary Data $^{1}$. Studies that did not provide clear data about COVID-19 characteristics in children and adolescents were excluded. Regardless of the small sample, the study by Han et al. was included in this category as it did not provide personalized details about the patients and could not be considered as a case report. Most studies derived from China, with one study from the USA. The percentage of males ranged between $54.5 \%$ and $63.9 \%$, while mean age ranged from 2 to 11 years. Comorbidities were observed more commonly in these studies, although in small percentages, except for the series of Xia et al. (35\% observed comorbidities) (Xia et al., 2020). Symptoms, laboratory findings, treatments, and outcomes are summarized in Table 2 in Supplementary Data $^{1}$. Fever was reported in the greater part of cases (ranging from $6.3 \%$ to $71.4 \%$ ), while radiological findings of pneumonia were also observed in most of them (rate ranging from $52.8 \%$ to $72.7 \%$ ). Other laboratory findings were relatively normal, expect for the series of Xia et al., where CRP was elevated in $35 \%$ of the cases and procalcitonin in $80 \%$; in this study, other pathogens such as Mycoplasma pneumoniae (12\%), influenza virus type B (8\%), and Enterobacter aerogenes (8\%) were also identified (Xia et al., 2020). Diarrhea was also a common symptom with rates up to $57.1 \%$ while Han et al. reported that digestive tract symptoms were more common in children than adults $(\mathrm{p}=0.012)$ (Han et al., 2020). Additionally, elevated AST and ALT levels were also recorded as in case series (Table 2 in Supplementary Data $^{1}$ ). There were a few data about treatment ( 3 out of 7 studies), with antiviral therapy and interferon being the most common, with rates up to $100 \%$ of the pediatric patients. No complications were observed in the cohort studies but one case of death was reported, in a 10-month-old child with intussusception who presented multi-organ failure and died 4 weeks after admission ( $\mathrm{Lu}$ et al., 2020); the gender or other details of the deceased child were not reported. Three more deaths are recorded in the case series from the USA, but COVID-19 was not confirmed as the likely cause of death, without providing further information about the latter. Admission to ICU also showed a low rate (0.6-1.8\%), while almost all patients presented full recovery.

\section{Discussion}

COVID-19 targets mostly adults, with the majority of cases being in the age group between 30 and 79 years old (Chang et al., 2020). Children and adolescents under 18 years old represent less than $5 \%$ of cases, a lot of them being asymptomatic (Ludvigsson, 2020). The pandemic led to a great number of reports, mostly referring to adults and presenting insufficient data about children. The descriptive and detailed analysis of pediatric COVID-19 is essential to address special treatment needs and avoid complications in children and adolescents.

A total of 33 case reports and small case series were retrieved in this systematic review. These studies provided sufficient patient data and were subjected to pooled analysis. Additionally, a total of 7 cohort studies were identified, which did not present data at an individual level, but their findings confirmed the results of case reports. Considering the 33 studies, the pooled sample presented a wide age range (between 2 days and 18 years); on the other hand in cohort studies mean age ranged from 2 to 11 years, while male gender slightly predominated (54.5-63.9\%).

According to our systematic review and pooled analysis of 33 studies, fever was the most frequent symptom in pediatric patients with an incidence of $54.2 \%$ Asymptomatic patients, while not rare, were not the majority of reported cases $(28.4 \%, 33 / 116)$, as described in previous studies (Castagnoli et al., 2020; Chang et al., 2020; Ludvigsson, 2020). There was a trend $(\mathrm{p}=0.08)$ towards male sex among asymptomatic cases, which was an interesting finding as male sex in adults is considered a risk factor. Diarrhea was the most frequent symptom (11\%) after upper respiratory tract symptoms, a pattern that confirms previous reports (Castagnoli et al., 2020; Chang et al., 2020; Ludvigsson, 2020); accordingly, Han et al. reported a greater prevalence of digestive tract symptoms in children, compared to adults.

A high prevalence of radiological findings suggestive of pneumonia was noted in cohort studies, ranging between $52.8 \%$ and $72.7 \%$ (Table 2 in Supplementary Data $\left.^{1}\right)$. Such findings were also observed in more than half of case reports while the pooled analysis revealed that $40.7 \%$ of asymptomatic cases might have pneumonia on radiologic exams (pooled NPV $=59.3 \%$ (95\%CI: 38.8-77.6\%). This is an important observation that raises the question whether all positive pediatric patients need radiologic examination, a strategy that has been proposed by other authors as well (Ludvigsson, 2020; Shen and Yang, 2020), but not widely adopted as COVID-19 associated pneumonia seems to be mild in children. 
The presence of radiologic pneumonia was related to age, as preschoolers were found to develop pneumonia findings more frequently, with a 6-fold increase in odds of positive radiological signs of pneumonia. Fever (sensitivity: 60.3\%, specificity: $48.8 \%$ ) showed the highest sensitivity, followed by (sensitivity $47.4 \%$, specificity: 76.7\%), rhinorrhea (sensitivity: $21.1 \%$, specificity: $88.4 \%$ ) and diarrhea (sensitivity: 10.3\%, specificity: $88.4 \%$ ) in the diagnosis of radiologic pneumonia. Additionally, radiological findings of pneumonia were strongly associated with admission to ICU. This may suggest that presence of radiological/CT lesions in the lungs could be used as an indicator to monitor pediatric patients more closely.

Other laboratory findings did not seem to be affected in the vast majority of case reports and case series. The findings in cohort studies were similar, except for the series of Xia et al., which presented high levels of co-infection with other pathogens. Elevated AST and ALT levels were also recorded in both sets of studies, a fact that should be taken into consideration.

An interesting remark pertains to the fact that administered treatments did not seem to follow any published recommendations (Shen and Yang, 2020); different drugs were administered, regardless of the presence or absence of pneumonia radiological signs. Interferon in the form of nebulization was widely administered in both types of studies; nevertheless, none of the retrieved studies clarified whether the treatment had any effect upon the infection outcome. No complication or delay in the recovery was recorded in cases where no treatment was given. Additionally, no clear guidelines for hospitalization existed, as even asymptomatic children were hospitalized (24.6\% of case series). About $90 \%$ of the cases had complete recovery, regardless of hospitalization or treatment administration. Thus, there is a need of further longitudinal studies, considering therapy management and guidelines.

Even if pediatric patients seem to have better outcomes, a small percentage was admitted in ICU $(8 \%$ in case reports/case series and $0.6 \%-1.8 \%$ in cohort studies). Most recently, eight children's cases were described in the UK that were transferred to ICU presenting a Toxic Shock Syndrome (TSS)- Kawasakilike inflammatory syndrome; four of them had confirmed family exposure to the novel coronavirus but only two of them were tested positive for SARS-CoV-2 (one of them post-mortem) (Riphagen et al., 2020). Additionally, an increase was observed in inflammatory syndromes and TSS in many countries, not directly linked to COVID-19, but raising awareness (ECDPC, 2020). Two of the ICU patients in our systematic review (Sun et al., 2020) were reported with septic shock syndrome. No complications were observed in the cohort studies and full recovery was mentioned in both case series and cohort studies. It should be noted that all patients who required ICU admission in the case series presented tachypnea, which should be considered as a manifestation of more severe disease. Therefore, pediatricians should keep in mind that severe complications of COVID-19, while rare, may eventually develop.

None of the included case reports and case studies has recorded any deaths. Only one case of death was retrieved from the cohort studies, referring to a 10-month-old child with intussusception ( $\mathrm{Lu}$ et al., 2020). Three deaths were reported in the series from USA (CDC, 2020) but COVID-19 was not confirmed as the likely cause of death. Infection fatality ratio of COVID-19 under 9 years old is estimated to be $0.00016 \%$, and $0.00695 \%$ in age groups between 10 and 19 years old (Verity et al., 2020).

Most studies from China followed a classification severity system with five classes and a modified one with four classes, according to officially published guidelines in China. Han et al. additionally classified pneumonia into mild (mild pneumonia, asymptomatic infection) and severe. No other specific severity stratification systems for children and adolescents with COVID-19 were recorded in the remaining studies.

Commenting on the limitations of the study, due to the emergency of the subject, the research period was brief, a problem also encountered in previous studies (Castagnoli et al., 2020). Most reports did not provide detailed data, leading to exclusion and reduction of the sample size. Numerous case series and studies were published at the outset of the pandemic and the presence of overlapping individuals in the populations under study cannot be totally ruled out. Language restrictions may also have limited the pool of eligible reports. Another inherent limitation of the pooled analysis pertains to the fact that published case reports/case series are subject to considerable selection bias, as they may represent a subset of more severe cases, seeking treatment in health services.

On the other hand, strengths of this systematic review and pooled analysis include the larger study sample, the separation of case reports and small case series from larger cohort studies, and the inclusion of a substantial proportion of cases from Europe and the USA, while previous reviews contained almost exclusively reports from China.

In conclusion, this systematic review and pooled analysis, highlighted fever as the most frequently reported clinical finding in pediatric COVID-19, followed by cough, rhinorrhea, and diarrhea. Asymptomatic cases, although not rare, were not the majority, whereas male gender might be weakly associated with asymptomatic status. Positive radiological findings of pneumonia were recorded in more than half of the patients, with higher prevalence in preschoolers. Interestingly, radiological lesions were prevalent in patients requiring ICUtreatment and $40.7 \%$ of asymptomatic cases had pneumonia on radiologic examination. This may suggest that even if many pediatric patients are asymptomatic, they still need close monitoring. Another interesting observation was that rhinorrhea and diarrhea present high specificity in cases with positive radiological findings, while tachypnea may be considered as a predictor of severity. Concerning 
the outcome, our study confirms accepted wisdom that pediatric patients tend to have full recovery and rarely present complications, while there is need for a thorough reassessment of treatment and management guidelines.

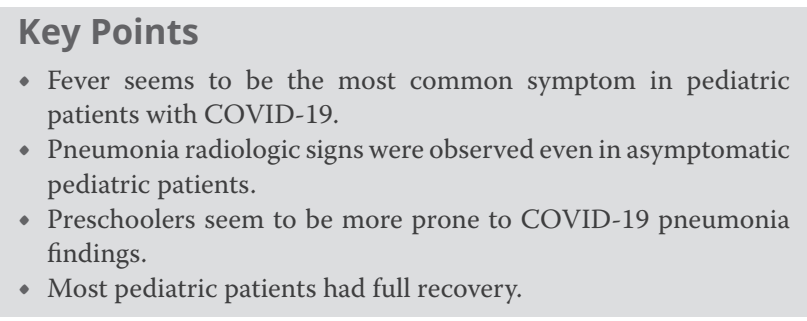

\section{References}

1. An P, Zhang M. (2020). Novel coronavirus SARS-CoV-2: familial spread resulting in COVID-19 pneumonia in a pediatric patient. Diagn Interv Radiol 26, 262-263 http://dx.doi.org/10.5152/ dir.2020.20157

2. Cai J, Xu J, Lin D, Yang Z, Xu L, et al. (2020). A Case Series of children with 2019 novel coronavirus infection: clinical and epidemiological features. Clin Infect Dis In press. http://dx.doi. org $/ 10.1093 / \mathrm{cid} /$ ciaa198

3. Canarutto D, Priolo A, Russo G, Pitea M, Vigone MC, Barera G. (2020). COVID-19 infection in a paucisymptomatic infant Raising the index of suspicion in epidemic settings. Pediatr Pulmonol 55 E4-E5. http://dx.doi.org/10.1002/ppul.24754

4. Castagnoli R, Votto M, Licari A, Brambilla I, Bruno R, et al. (2020). Severe Acute Respiratory Syndrome Coronavirus 2 (SARS-CoV-2) Infection in Children and Adolescents: A Systematic Review. JAMA Pediatr In press. http://dx.doi org/10.1001/jamapediatrics.2020.1467.

5. Chan JF, Yuan S, Kok KH, To KK, Chu H, et al. (2020). A familial cluster of pneumonia associated with the 2019 novel coronavirus indicating person-to-person transmission: a study of a family cluster. Lancet 395, 514-523. http://dx.doi.org/10.1016/s01406736(20)30154-9

6. Chang TH, Wu JL, Chang LY. (2020). Clinical characteristics and diagnostic challenges of pediatric COVID-19: A systematic review and meta-analysis. J Formos Med Assoc 119, 982-989. http://dx.doi.org/10.1016/i.jfma.2020.04.007

7. Chen ZM, Fu JF, Shu Q, Chen YH, Hua CZ, et al. (2020). Diagnosis and treatment recommendations for pediatric respiratory infection caused by the 2019 novel coronavirus. World J Pediatr 16, 240-246. http://dx.doi.org/10.1007/s12519-020-00345-5

8. CDC COVID-19 Response Team. Coronavirus Disease 2019 in Children - United States, February 12-April 2, 2020, (2020) MMWR Morb Mortal Wkly Rep 69, 422-426. http://dx.doi. org/10.15585/mmwr.mm6914e4

9. Cui Y, Tian M, Huang D, Wang X, Huang Y, et al. (2020). A 55-Day-Old Female Infant infected with COVID 19: presenting with pneumonia, liver injury, and heart damage. J Infect Dis 221, 1785-1781. http://dx.doi.org/10.1093/infdis/jiaa113

10. Dong Y, Mo X, Hu Y, Qi X, Jiang F, Jiang Z, Tong S. (2020). Epidemiology of COVID-19 Among Children in China. Pediatrics In press. http://dx.doi.org/10.1542/peds.2020-0702

11. European Centre for Disease Prevention and Control (2020). Paediatric inflammatory multisystem syndrome and SARS$\mathrm{CoV}-2$ infection in children.

12. Han YN, Feng ZW, Sun LN, Ren XX, Wang H et al. (2020). A comparative-descriptive analysis of clinical characteristics in 2019-coronavirus-infected children and adults. Journal of medical virology. 92, $1596-1602$. http://dx.doi.org/10.1002/ jmv. 25835

13. Hu Y, Sun J, Dai Z, Deng H, Li X (2020). Prevalence and severity of corona virus disease 2019 (COVID-19): A systematic review and meta-analysis. J Clin Virol 127, 104371. http://dx.doi org/10.1016/j.jcv.2020.104371
14. Hu Z, Song C, Xu C, Jin G, Chen Y, et al (2020). Clinical characteristics of 24 asymptomatic infections with COVID-19 screened among close contacts in Nanjing, China. Sci China Life Sci 63, 706-711. http://dx.doi.org/10.1007/s11427-020-1661-4

15. Huang L, Zhang X, Zhang X, Wei Z, Zhang L. (2020). Rapid asymptomatic transmission of COVID-19 during the incubation period demonstrating strong infectivity in a cluster of youngsters aged 16-23 years outside Wuhan and characteristics of young patients with COVID-19: A prospective contact-tracing study. J Infect 80, e1-e13. http://dx.doi.org/10.1016/j.jinf.2020.03.006

16. Ji LN, Chao S, Wang YJ, Li XJ, Mu XD et al. (2020). Clinical features of pediatric patients with COVID-19: a report of two family cluster cases. World J Pediatr In press http://dx.doi. org/10.1007/s12519-020-00356-2

17. Kam KQ, Yung CF, Cui L, Lin Tzer Pin, Mak TM (2020). A Well Infant with Coronavirus Disease 2019 (COVID-19) with High Viral Load. Clin Infect Dis 71, 847-849. http://dx.doi.org/10.1093/ cid/ciaa201

18. Kanne JP (2020). Chest CT Findings in 2019 Novel Coronavirus (2019-nCoV) Infections from Wuhan, China: Key Points for the Radiologist. Radiology 295, 16-17. http://dx.doi.org/10.1148/ radiol.2020200241

19. Le HT, Nguyen LV, Tran DM, Do HT, Tran HT et al. (2020). The first infant case of COVID-19 acquired from a secondary transmission in Vietnam. Lancet Child Adolesc Health 4, 405406. http://dx.doi.org/10.1016/s2352-4642(20)30091-2

20. Li B, Shen J, Li L., Yu C. (2020). Radiographic and Clinical Features of Children with 2019 Novel Coronavirus (COVID-19) Pneumonia. Indian Pediatr In press.

21. Li W, Cui H, Li K, Fang Y, Li S (2020). Chest computed tomography in children with COVID-19 respiratory infection. Pediatr Radiol 50, 796-799. http://dx.doi.org/1001007/s00247-020-04656-7.

22. Li Y, Guo F (2020). Insight into COVID-2019 for pediatricians. Pediatr Pulmonol 55, E1-e4. http://dx.doi.org/10.1002/ ppul.24734

23. Liberati A, Altman DG, Tetzlaff J, Mulrow C, Gøtzsche PC, et al. (2009). The PRISMA statement for reporting systematic reviews and meta-analyses of studies that evaluate health care interventions: explanation and elaboration. J Clin Epidemiol 62, e1-34.

24. Lin J, Duan J, Tan T, Fu Z, Dai J. (2020). The isolation period should be longer: Lesson from a child infected with SARS-CoV-2 in Chongqing, China. Pediatr Pulmonol. 55, E6-E9. http://dx.doi. org/10.1002/ppul.24763

25. Liu H, Liu F, Li J, Zhang T, Wang D, Lan W. (2020). Clinical and CT imaging features of the COVID-19 pneumonia: Focus on pregnant women and children. J Infect 80, e7-e13. http://dx.doi. org/10.1016/j.jinf.2020.03.007

26. Liu M, Song Z, Xiao K. (2020). High-Resolution Computed Tomography Manifestations of 5 Pediatric Patients With 2019 Novel Coronavirus. J Comput Assist Tomogr 3, 311-313. http:// dx.doi.org/10.1097/rct.00000000000001023

27. Liu W, Zhang Q, Chen J, Xiang R, Song H. (2020). Detection of Covid-19 in Children in Early January 2020 in Wuhan, China. New England Journal of Medicine. 382, 1370-1371. http://dx.doi. org/10.1056/NEJMc2003717

28. Liu Y, Yang Y, Zhang C, Huang F, Wang F. et al. (2020). Clinical and biochemical indexes from 2019-nCoV infected patients linked to viral loads and lung injury. Sci China Life Sci 63, 364374. http://dx.doi.org/10.1007/s11427-020-1643-8

29. Lou XX, Shi CX, Zhou CC, Tian YS (2020). Three children who recovered from novel coronavirus 2019 pneumonia. J Paediatr Child Health 56, 650-651. http://dx.doi.org/10.1111/jpc.14871

30. Lu X, Zhang L, Du H, Zhang J, Li YY et al. (2020). SARS-CoV-2 Infection in Children. New England Journal of Medicine 382, 1663-1665. http://dx.doi.org/10.1056/NEJMc2005073

31. Ludvigsson JF (2020). Systematic review of COVID-19 in children shows milder cases and a better prognosis than adults. Acta Paediatr 6, 1085-1088. http://dx.doi.org/10.1111/apa.15270 
32. Ma X, Su L, Zhang Y, Zhang X, Gai Z, Zhang Z. (2020). Do children need a longer time to shed SARS-CoV-2 in stool than adults? J Microbiol Immunol Infect 3, 373-376. http://dx.doi. org/10.1016/j.jmii.2020.03.010

33. Pan X, Chen D, Xia Y, Wu X, Li T et al. (2020). Asymptomatic cases in a family cluster with SARS-CoV-2 infection. Lancet Infect Dis 20, 410-411. http://dx.doi.org/10.1016/s14733099(20)30114-6

34. Park JY, Han MS, Park KU, Kim JY, Choi EH (2020). First Pediatric Case of Coronavirus Disease 2019 in Korea. J Korean Med Sci 35, e124. http://dx.doi.org/10.3346/jkms.2020.35.e124

35. Qian G, Yang N, Ma AHY, Wang L, Li G et l. (2020). A COVID-19 Transmission within a family cluster by presymptomatic infectors in China. Clin Infect Dis 15, 861-862. http://dx.doi.org/10.1093/ cid/ciaa316

36. Qiu H, Wu . (J, Hong L, Luo Y, Song Q, Chen D. (2020). Clinical and epidemiological features of 36 children with coronavirus disease 2019 (COVID-19) in Zhejiang, China: an observational cohort study. Lancet Infect Dis 6, 689-696. http://dx.doi org/10.1016/s1473-3099(20)30198-5

37. Riphagen S, Gomez X, Gonzalez-Martinez C, Wilkinson N, Theocharis P. (2020). Hyperinflammatory shock in children during COVID-19 pandemic. Lancet Lond Engl 10237, 1607 1608. http://dx.doi.org/10.1016/S0140-6736(20)31094-1

38. Robbins E, Ilahi Z, Roth P. (2020). Febrile Infant: COVID-19 in Addition to the Usual Suspects. Pediatr Infect J 6, e81-e82. http:// dx.doi.org/10.1097/inf.0000000000002693

39. Shen K, Yang Y, Wang T, Zhao D, Jiang Y et al. (2020). Diagnosis, treatment, and prevention of 2019 novel coronavirus infection in children: experts' consensus statement. World J Pediatr 3, 223231. http://dx.doi.org/10.1007/s12519-020-00343-7.

40. Shen KL, Yang YH (2020). Diagnosis and treatment of 2019 novel coronavirus infection in children: a pressing issue. World J Pediatr 3, 219-221. http://dx.doi.org/10.1007/s12519-020-00344-6

41. Shen Q, Guo W, Guo T, Li J, He W, et al. (2020). Novel coronavirus infection in children outside of Wuhan, China. Pediatric Pneumonology 6, 1424-1429. http://dx.doi.org/10.1002/ ppul.24762

42. Šimundić AM (2009). Measures of Diagnostic Accuracy: BasicDefinitions. EJIFCC 19, 203-211

43. Stang A. (2020). Critical evaluation of the Newcastle-Ottawa scale for the assessment of the quality of nonrandomized studies in meta-analyses. European Journal of Epidemiology 25, 603605. http://dx.doi.org/10.1007/s10654-010-9491-z

44. Sun D, Li H, Lu XX, Xiao H, Ren J et al. (2020). Clinical features of severe pediatric patients with coronavirus disease 2019 in Wuhan: a single center's observational study. World J Pediatr. 3, 251-259. http://dx.doi.org/10.1007/s12519-020-00354-4.
45. Tang A, Tong ZD, Wang HL, Dai YX, et al. (2020). Detection of Novel Coronavirus by RT-PCR in Stool Specimen from Asymptomatic Child, China. Emerg Infect Dis 26 1337-1339. http://dx.doi.org/10.3201/eid2606.200301

46. Verity R, Okell LC, Dorigatti I, Winskill P, Whittaker C et al. (2020). Estimates of the severity of coronavirus disease 2019: a model-based analysis. Lancet Infect Dis 6, 669-677. http:/! dx.doi.org/10.1016/s1473-3099(20)30243-7

47. Wang S, Guo L, Chen L, Liu W, Cao Y et al. (2020). A case report of neonatal COVID-19 infection in China. Clin Infect Dis 15, 853-857. http://dx.doi.org/10.1093/cid/ciaa225

48. Wei M, Yuan J, Liu Y, Fu T, Yu X, Zhang, ZJ. (2020). Novel Coronavirus Infection in Hospitalized Infants Under 1 Year of Age in China. Jama 13, 1013. http://dx.doi.org/10.1001/ jama.2020.2131

49. WHO, (2020) World Health Organization [WWW Document] Coronavirus Dis. COVID-19 Outbreak Situat.

50. Xia W, Shao J, Guo Y, Peng X, Li Z et al. (2020). Clinical and CT features in pediatric patients with COVID-19 infection: Different points from adults Pediatr Pulmonol 55, 1169-1174. http:/! dx.doi.org/10.1002/ppul.24718

51. Xing YH, Ni W, Wu Q, Li WJ, Li GJ et al. (2020). Prolonged viral shedding in feces of pediatric patients with coronavirus disease 2019. J Microbiol Immunol Infect 3, 473-480. http://dx.doi. org/10.1016/j.jmii.2020.03.021

52. Xu R, Du M, Li L, Zhen Z, Wang H, Hu X. (2020). CT imaging of one extended family cluster of corona virus disease 2019 (COVID-19) including adolescent patients and "silent infection." Quant Imaging Med Surg 10, 800-804. http://dx.doi. org/10.21037/qims.2020.02.13

53. Xu Y, Li X, Zhu B, Liang H, Fang C et al. (2020). Characteristics of pediatric SARS-CoV-2 infection and potential evidence for persistent fecal viral shedding. Nature Medicine 26, 502-505. http://dx.doi.org/10.1038/s41591-020-0817-4

54. Zhang T, Cui X, Zhao X, Wang J, Zheng J et al. (2020). Detectable SARS-CoV-2 viral RNA in feces of three children during recovery period of COVID-19 pneumonia. J Med Virol 92, 909-914. http:// dx.doi.org/10.1002/jmv.25795

55. Zheng F, Liao C, Fan QH, Chen HB, Zhao XG, Xie ZG et al (2020). Clinical Characteristics of Children with Coronavirus Disease 2019 in Hubei, China. Curr Med Sci 40, 275-280. http://dx.doi. org/10.1007/s11596-020-2172-6

56. Zhu L, Wang J, Huang R, Liu L, Zhao H, Wu C. (2020). Clinical characteristics of a case series of children with coronavirus disease 2019. Pediatr Pulmono 55, 1430-1432 http://dx.doi. org/10.1002/ppul.24767

57. Zhu, N, Zhang D, Wang W, Li X, Yang B et al. (2020). A Novel Coronavirus from Patients with Pneumonia in China, 2019. N Engl J Med 382, 727-733. http://dx.doi.org/10.1056/NEJMoa2001017 